\title{
Positioning as Service for 5G IoT Networks
}

Brahim El Boudanli, Student Member, IEEE, Loizos Kanaris ${ }^{2}$, Senior Member, IEEE, Akis Kokkinis ${ }^{2}$, Senior Member, IEEE, Christos Chrysoulas ${ }^{3}$, Senior Member, IEEE, Tasos Dagiuklas ${ }^{1}$, Senior Member, IEEE Stavros Stavrou $^{4}$, Senior Member, IEEE

Department of Computer Science and Informatics, London South Bank University, UK ${ }^{1}$ Sigint Solutions Ltd, 10-12, Demokedous street, $1^{\text {st }}$ floor, Nicosia 2311, Cyprus ${ }^{2}$

School of Computing, Edinburgh Napier University, 10 Colinton Rd, Edinburgh, EH10 5DT, Scotland, U.K. ${ }^{3}$

Faculty of Pure and Applied Sciences, Open University of Cyprus, 2220 Nicosia, Cyprus ${ }^{4}$

\begin{abstract}
Big Data and Artificial Intelligence are new technologies to improve indoor localization. It focuses on the use of machine learning probabilistic algorithms to extract, model and analyse live and historical signal data obtained from several sources. In this respect, the data generated by $5 \mathrm{G}$ network and the Internet of Things is quintessential for precise indoor positioning in complex building environments. In this paper, we present a new architecture for assets and personnel location management in 5G network with an emphasis on vertical sectors in smart cities. Moreover, we explain how Big Data and Machine learning can be used to offer positioning as service. Additionally, we implement a new deep learning model for 3D positioning using the proposed architecture. The performance of the proposed model is compared against other Machine Learning algorithms.
\end{abstract}

Index Terms-Indoor Positioning, Internet of Things, 5G, Deep Learning, Big Data, RSS, Radiomap

\section{INTRODUCTION}

$\mathbf{T}$ He 5G NR (New Radio Technologies) and IoT (Internet of Things) are indispensable for the provisioning of improved decentralized distributed services across smart cities. Propped up by AI (Artificial Intelligence) and Big Data, several vertical sectors witnessed waves of radical changes aiming to transform the way in which core processes are conducted. According to Cisco, IoT technologies are expected to be bandwidth and traffic-intensive reaching 3.9 billion connected M2M devices by 2022 [1]. It is undeniable that these breakthroughs are very beneficial to the several vertical sectors. However, little has been done to address the issue of positioning in smart cities with massively deployed IoT networks. A recent 5GPPP technical report set out detailed positioning requirements for vertical sectors such as: Smart manufacturing, transportation and e-agriculture[2]. The report

discusses context-aware applications that should offer realtime tracking for valuable assets in factories like UAV (Unmanned Ariel Vehicles), AGV (Automated Guided Vehicles) and other geo-tagged expensive goods. Position-tracking systems are expected to perform around 1 meter accuracy in 3D environment and must rely on existing and emerging technologies like WiFi 6 and WiFi 7 wireless networks, BLE (Bluetooth Low Energy) and WSN (Wireless Sensors Network). In this paper, we propose an architecture for multiple tracked tenants in 5G IoT network using Machine Learning and Big Data. We discuss an implementation of the Deep Learning 3D positioning model for tracking assets and people based on the suggested architecture. The rest of this paper is structured as follow: Section II covers current research status and our contributions. Section III describes a five tier architecture for positioning concept. Section IV explains the different functionalities of the proposed knowledge plane. Section IV-F deals with a 3D positioning algorithm implemented on 5G emulated environment. Section V summarizes the findings and future work.

\section{State of The ART}

Indoor positioning in 5G IoT networks is still a very new research area. It involves making use of new emerging radio technologies to improve the location accuracy. Michal et al.[3] have proposed an architecture for real-time location tracking using information fusion from both Wi-Fi and dead reckoning sources. However, since the current position of the tracked is calculated using the previous location, the distance error becomes cumulative and grows over the time. Moreover, this system does not offer 3D positioning which critical nowadays for indoor settings. Additionally, it suffers from attenuation and additional hardware is always required to support the accuracy. Therefore, it is not effective for tracking multiple agents in complex environments. on the concept of data fusion can be found on [4]. Similarly, to recognise the item location inside a warehouse, authors in [5] a passive radio-frequency localization system which utilises passive RFID (Radio-frequency identification). This solution is costly to setup and maintain especially for mega-warehouses and massively stored assets. Another interesting system for positioning called SnapLoc has been presented in [6]. Authors have implemented UWB (Ultra Wide-Band) system which they claim to be scalable to unlimited tags. However, UWB is known for slower adoption are, high cost for implementation

and signal penetration especially inside complex buildings [7]. A survey of similar applications and system can be found in [8]. Despite the existence of encouraging works in the literature like [9] and [10], most of the existing literature does not high-light the role of $5 \mathrm{G}$, machine learning and big data to cater for massive indoor positioning. This is one of the motivations for proposing this architecture. The second motive is the lack of 3D positioning for complex environments. In this research paper we contribute with following:

- A solid scalable and expandable architecture for decentralized positioning in $5 \mathrm{G}$ enabled environment such as warehouses, malls and factories.

- A big data and machine learning enabled positioning for continuous improved learning. 


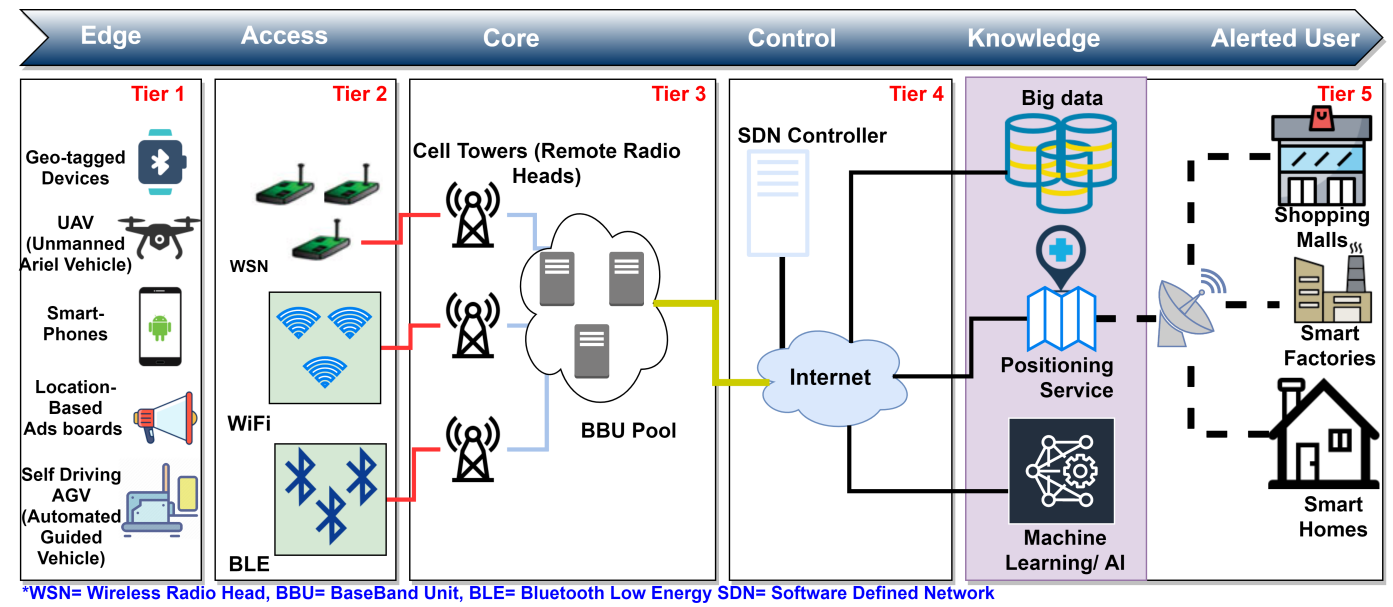

Fig. 1. Positioning as a Service for 5G IoT networks

- An implementation use case of vertical and horizontal positioning model for massively deployed positioning in complex buildings.

The following section covers in details these elements.

\section{Five Tiers Architecture for 5G SMARt cities}

There is no doubt that $5 \mathrm{G}$ is going to connect IoT device and transform dummy equipment into smart ones. However, smart warehouses, malls and factories are currently facing major challenges managing their assets especially when they are scattered around the entire sites unattended. To prevent these scenarios from happening, we propose an architecture for positioning as a service for complex environment. In this section, a five tier novel architecture is presented for location management using Big Data and machine learning. Figure 1 depicts different components of this architecture. These different components belong to different planes as shown in Figure 2.

\section{A. Data Plane}

The Data plane, as suggested by 5GPPP [2], works by decoupling the hardware and software components. From an SDN (Software Defined Network) prospective, the aim of this separation is to move certain network functionalities to a distributed softwarized subsystems. Thus, the softwarized programmable networks can be realized. The new 5G C-RAN architecture is one example of this decoupling concept where several RRH (Remote Radio Heads) antennas are deployed and interconnected to a single BBU (Base Band Unit). This plane provides data links to connected network hosts.It consists of a set of hardware and software nodes. A software node can either be a virtual switch like Open Vswitch [11] or a cloudbased BBU (Base Band Unit) while a hardware node can be a C-RAN (Cloud-Radio Access Network) RRH antenna, a WSN (Wireless Sensor Network), a WiFi 6 hotpot, a BAN (Body Area Network). In Figure 2, the data plane represents all elements from tier one to tier three illustrated in Figure 1.
1) Network Edge: : the network edge slice compromises of network connected hosts such as inventory scanning devices, indoor operating vehicles(Autonomous and semi-autonomous) and tagged personnel. The tracked devices usually come with RFID, WiFi or BLE capabilities enabled in them. The slice acts as a signal transmitter for the positioning service in the knowledge plane.

2) Network Access: In the second tier, network access consists of a set of networks such as Wireless Sensors (temperatures, humidity etc.), BLE and WiFi Networks. These Wireless networks bands in $5 \mathrm{G}$ are expected to range between $1 \mathrm{Ghz}$ and $6 \mathrm{Ghz}$. These technologies can either be used together or separately during the radio planning process of the positioning. It is suggested a combination of two or three from these technologies to get better accuracy in [4].

3) Network Core: The third tier consists of 5G Cloud Radio Access Network. This a unique 5G concept divides the Radio Access Network into two separate entities: an RRH antenna and BBU unit placed on the cloud. An implementation of this concept is mentioned in Section IV-F.

\section{B. Control Plane}

This plane, as proposed by 5GPPP [2], includes Software Defined controllers responsible for orchestrating the network and managing the packets flow. Usually, this plane is reserved for SDN controllers like OpenDaylight [12]. Its functionalities can be further expanded to include event-triggered geo-fencing options through out writing or blocking flows to a specific connected device based on previously defined geographical fences.

1) Network Control: The Software defined nature of $5 \mathrm{G}$ makes it necessary to have a control plane. Tier four includes an SDN (Software Defined Network) Controller responsible for managing network flows and packing matching and blocking. The role of this slice is to patrol the incoming and outgoing traffic in a $5 \mathrm{G}$ environment.

\section{Application Plane}

From the SDN architecture, an application plane includes application built on top control plane. As shown in Figure 2, 
we developed an application to collect signal data and aggregates them. The functionalities of this application are discussed further in Section IV.

\section{Knowledge Plane}

This plane has been added to the original architecture to serve the purpose of decentralized positioning. As depicted on Figure 2, this plane is made up of four components: Data Aggregation and Standardization Gate, Position Visualizer, Historical Big Data Aggregator and Machine Learning Engine.

1) Knowledge: The knowledge plane is a member of last tier. Tier five concerns the positioning service or the knowledge plane and the service/alerted users. The knowledge slice consists of three main components: Machine Learning/AI engine that conducts the modeling, a positioning service for both live and historical location tracking and a Big Data component to store both historical data and the positioning model parameters.

2) Alerted Users: The alerted users are the second members of tier 5. They are the system users and they have direct access to the positioning service but no direct access to the Machine Learning engine or the Big Data repository. The alerted users can be systems like a mall management system, a smart factory operation application or a smart home application. The next section covers the functional architecture of this framework and the different interactions between each component.

\section{KNOWLEDGE PLANE FRAMEWORK COMPONENTS:}

In this section, as previously mentioned, we have extended the original architecture introduced in [2]. Furthermore, we demonstrate the different components of the knowledge plane and their functionalities. The plane collects and store signal data from various $5 \mathrm{G}$ IoT sources using client collector to provide positioning as a service. Figure 2 illustrates the proposed plane along with its interconnections with data, control and application planes.

\section{A. Clients Collector}

This application is deployed on the UE (User-Equipment) on the edge of the network with the purpose of collecting wireless signal data and send it to the master collector periodically. It runs as a daemon while establishing a reliable secure shell connection with the Master Collector application. The main function of this component is sending RSS and other signal data to the Master Collector to be aggregated.

\section{B. Master Collector}

The Master Collector receives RSS signal data from several IoT application collectors. First, it establishes and maintains a reliable and secure (TCP, SSH) connection with one or multiple client collector on the Data plane. Second, it aggregates the fetched data and stores centrally for location estimation and visualization at the knowledge plane level.

\section{Data Aggregation and Standardization Gate}

An API (Application Programming Interface) platform has been created to service data in a structure manner to the different components of the knowledge plane. The platform has been created used Flask web-server on Python version 2.7. The API acts as a link between the application plane and the positioning knowledge plane. This component receives data via a POST request and serve the available data via a GET request.

\section{Machine Learning Engine}

This component performs data pre-processing, offline and online training. The end results is an accurate location for each tracked device. The tasks involved in this components are: Pre-processing, offline training, online training, positioning modeling.

- Pre-processing: In this sub-component, a staging area is created for the model the training units. It performs data acquisition, data quality checks and validations, imputing and standardization. Typically, $70 \%$ of the overall process time is spent on this phase.

- Offline Training: Once the pre-processing tasks are completed, the offline training starts by dividing the data into training, validation and testing for the machine learning model.

- Online Training: This sub-component validates the positioning accuracy of the model built during offline training. This can be in the form of real-time signal data fed from the client collector of the IoT devices.

- Position Modelling: positioning modelling constructs a model using a machine learning library such as Keras, Pytorch or Tensorflow [13]. It learns from the fed dataset, and generates a model for online position estimation.

\section{E. Historical Big Data Aggregator}

In this plane, Big Data supports two use cases. On one hand, it stores offline training and prediction performed by machine learning engine in a data repository. Network signal data collected from the Aggregator API are processed into a individual time-series and stored centrally. On the other hand, the repository provides the historical visualizer with timestamped locations of the devices connected to the positioning service. A Cloudera-HBase server is used for this purpose.

\section{F. Live/Historical Position Visualizer}

A web-based data visualization / dashboard tool has been developed to have a global view of assets tracking. The main two services offered are the following: live location and historical location of people and devices.

\section{G. Network Setup}

The network setup provided in this experiment consists of a set of hardware and software components put together to emulate an IoT network in $5 \mathrm{G}$ environment. The setup is used to leverage signal data for the purpose of 3D positioning. 
Positioning as a Service Functional Architecture for 5G Enabled IoT Networks

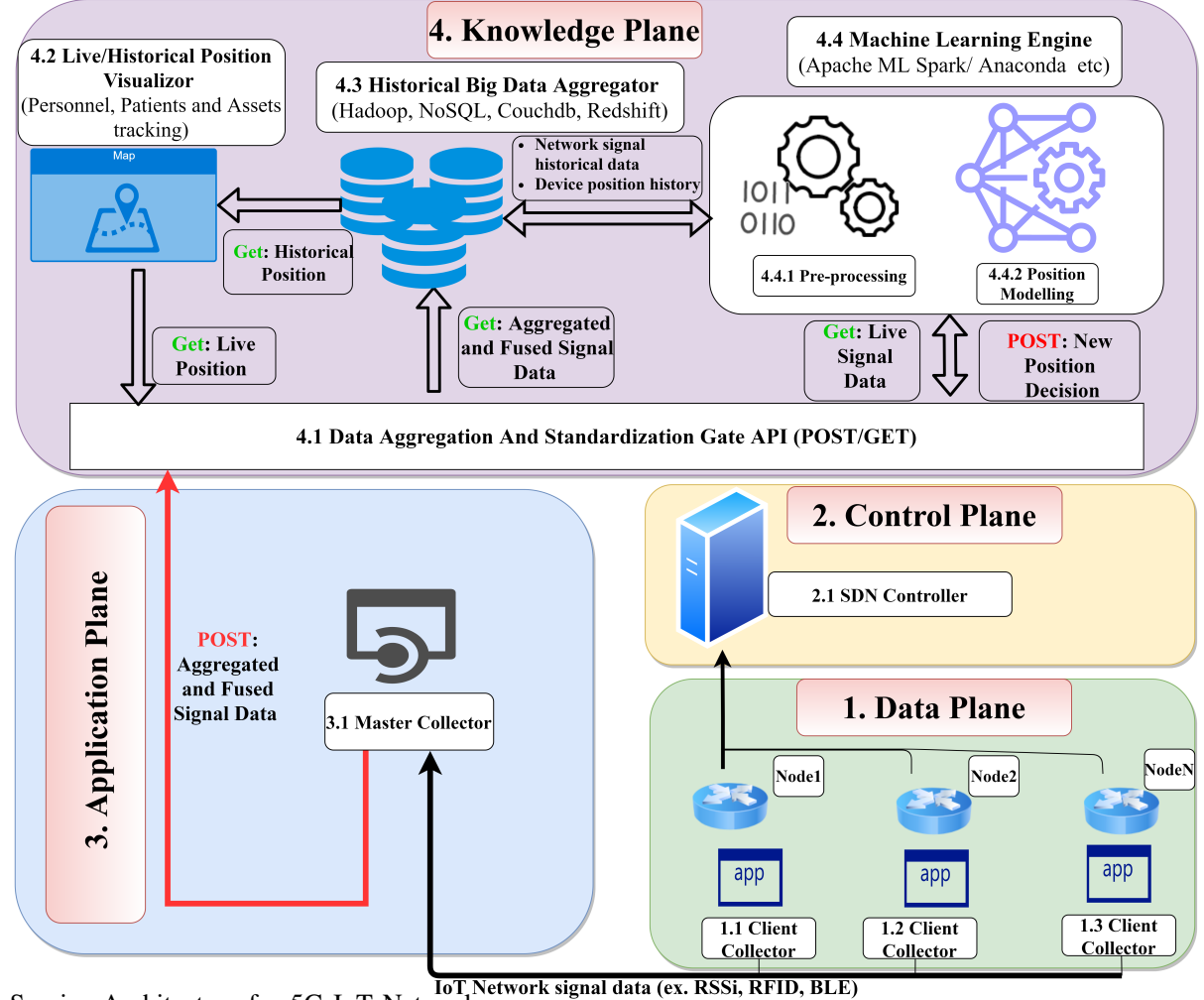

Fig. 2. Positioning as a Service Architecture for 5G IoT Network

1) $5 G$ Wireless IoT Network: In this test environment, we consider an outdoor to indoor $5 \mathrm{G}$ wireless network, emulated by typical IoT network with Zolertia RE-Mote Revision B nodes. This WSN is a typical network found in a smart buildings as illustrated in tier 2 Figure 1. We have deployed five nodes in this testbed. We have constructed a radiomap from this environment following the steps mentioned in [14]. The database of RSS signal with corresponding reference points have been used to create a positioning model.

2) $5 G C-R A N$ on GNS3 Emulator: 5G C-RAN is made up of two main components: A RRH and a BBU (BaseBand Unit). The former is responsible for handling the analogue signal processing functionality while the later performs digital packets processing. To build this concept, we have used GNS3 version 2.2.6 [15], an open source network emulator. The latter, as shown on Figure 3(a), the BBU component is placed on a cloud environment connecting it to the core network.

3) OpenDaylight Controller: The OpenDaylight [12] is a controller used to patrol the incoming and the outgoing traffic in a network. To make the testbed support a SDN (Software Defined Network), each node has Open vSwitch [11] installed on it. Each Open vSwitch on the C-RAN is connected to the OpenDaylight controller via the openflow port number 6633. Figure 3(b) shows how a group of 5 Zolertia REMote Revision B node is connected to the C-RAN in blue. The interface shown is from the controller topology view of OpenDaylight.

\section{H. $3 D$ positioning simulation}

The concept of Indoor Positioning can be implemented on various types of environments and usage scenarios involving both person and asset tracking in complex building environment. In this 3D positioning simulation, a DNN (Deep Neural Networks) and RSS fingerprint-based localization approach has been implemented in a 5G IoT setup testbed explained in previous section. Without loss of generality, we consider an indoor $5 \mathrm{G}$ wireless network, emulated by typical IoT network with Zolertia RE-Mote Revision B.

1) Data Collection: Our radiomap consists of $28803 \mathrm{D}$ reference points associated with RSS values from five different WSN Access Points(APs). The total number of features used to create the model is eight. The environment fingerprints radiomap is generated through the use of fully deterministic 3D simulator called TrueNet [16]. To ensure there is no redundancy in the information collected, we conducted a Pearson correlation test between each access point.

2) Data Pre-prossessing: Before going through modelling, the data collected during this experiment have to undergo certain pre-processing step. First, the vertical plane of the radio map represented by a column called $\mathrm{Z}$ in the radio map has three distinct values. These values have been transformed to three columns using One-hot-Encoding technique. The output columns are to be used later a target variables for the vertical location classification model. Second, to make the learning easier and faster for each model, the original values 
(a) 5G emulated C-RAN testbed on GNS3

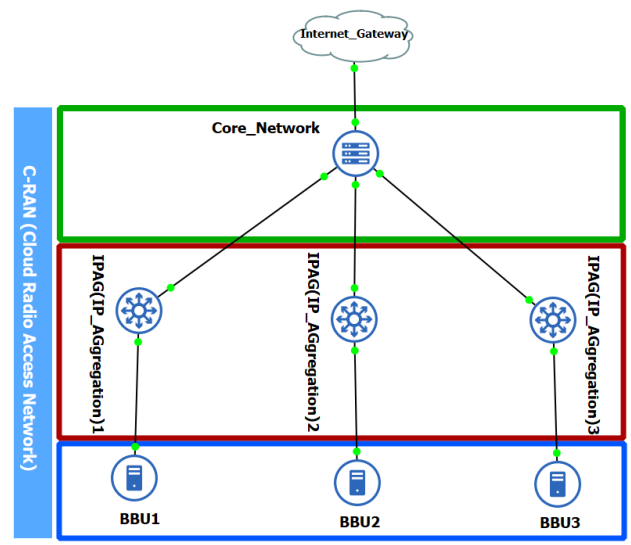

(b) WSN and GNS3 emulated $5 \mathrm{G}$ C-RAN

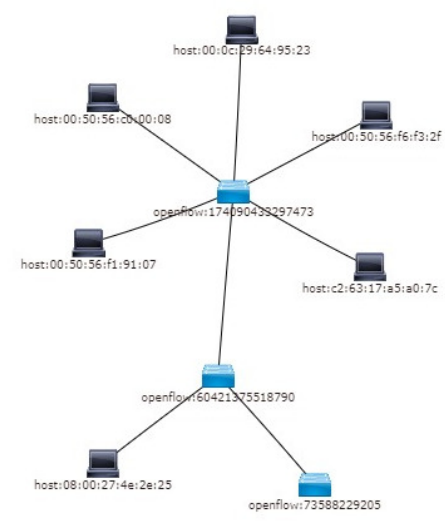

Fig. 3. 5G C-RAN setup on GNS3 and Wireless Sensor Network connected to OpenDaylight SDN controller

(a) Vertical positioning misclassification (b) Positioning model mean euclidean distance error in meter (m) vs the count for each Model

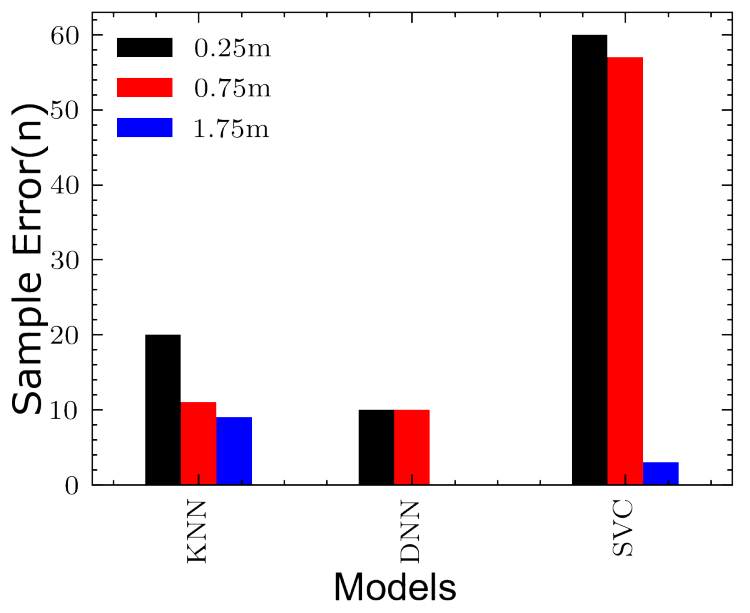
number of epochs

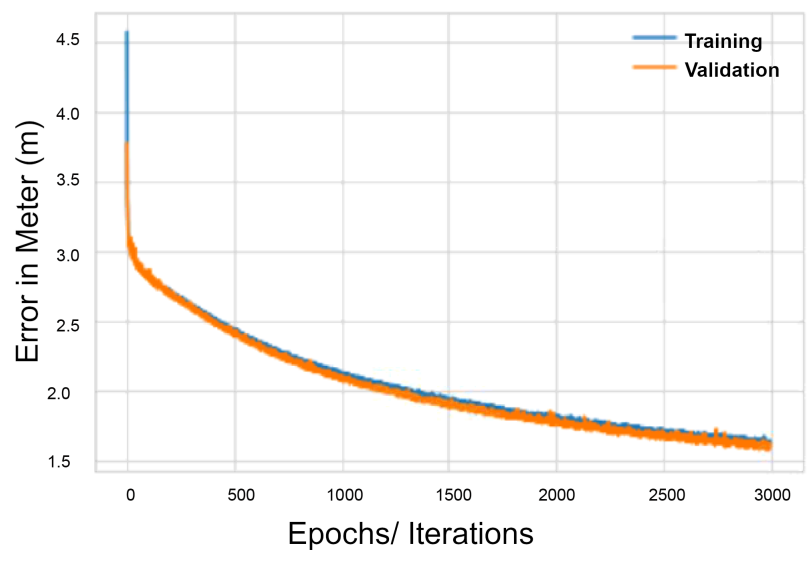

(c) 2D positioning model comparison: DNN VS KNN VS SVM
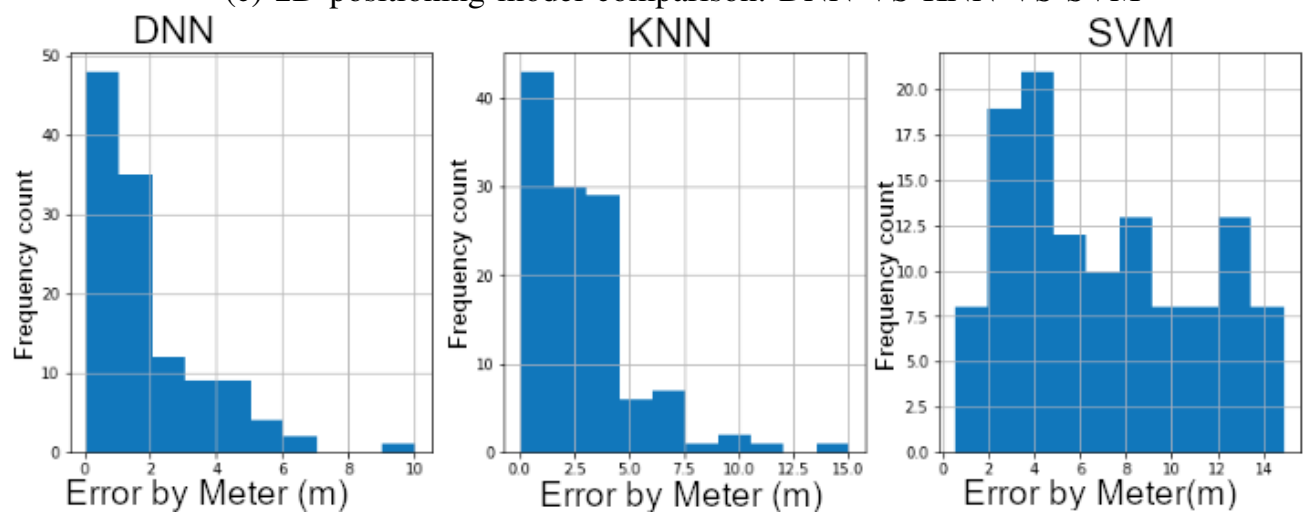

Fig. 4. 3D Positioning using 5G C-RAN and Wireless Sensors Network machine learning results

of each access points have been normalized using Min-Max normalization method.

3) Modelling: In this simulation, a multi-layered radiomap dataset on two DNN [13] models have been trained to accurately predict the $3 \mathrm{D}$ location of a user equipment. The first model consists of 3 hidden layers with 300 neurons each and two output variables $\mathrm{X}$ and $\mathrm{Y}$ location. The second neural network has also 3 hidden layers and 270 neurons but with 3 classes each representing a vertical height in meter $(0.25 \mathrm{~m}, 0.75 \mathrm{~m}$ and $1.75 \mathrm{~m})$. The two trained networks have been compared with industry used machine learning models KNN and Support Vertor Machine. The result analysis of this 
comparison is later discussed in the following subsections.

4) Off-line Training: During the off-line training, each model has been propped with a layer of batch normalization to speed up the process of the learning. Firstly, the optimization algorithm used to train both networks is ADAM (Adaptive Moment Estimation) [13]. Secondly, as illustrated in Figure4(b), to make sure each model is trained properly, the first model has been trained using MED( Mean Euclidean Distance) error cost function [13]. Finally, after 3000 epochs, the 2D model has reached a 1.6 meter MED error.

5) Results Analysis:

- 2D Positioning Model Analysis: Using 180 random samples [16], the proposed 2D Neural Network model is benchmarked against SVR (Support Vector Regression) and KNN (K-Nearest Neighbor). A full comparison is provided in Figure 4(c). SVR has scored a the highest error distribution where the peak of its distribution ranges between four and six meters error. KNN has performed slightly better compared to SVR. However, a large proportion of the distribution error falls between 3 and 5 meters, which makes it the second worse performing after SVR. The proposed 2D positioning model has given the best performance. The peak of its distribution error falls between zero and two meters with a mean error of $1.6 \mathrm{~m}$.

- Vertical Positioning Model Analysis: Using 180 random samples as previously, we estimated the vertical positioning of the UE. The results in Figure 4(a)shows a visual comparison of our proposed model (DNN), SVC ( Support Vector Classification) and KNN. Each model has been given an equal number of three classes $0.25 \mathrm{~m}$, $1.25 \mathrm{~m}$ and $1.75 \mathrm{~m}$. We have calculated the misclassification count for each of these models as shown in Figure 4(a). At first glance, Figure 9 shows that SVC has performed very badly in terms of classification of observations. The model has failed to accurately classify during the online phase. More than 66\% - circa 120 samples- were wrongly classified. With a total of 40 misclassified samples, KNN has performed better than SVC but still does not differentiate between certain classes properly. Our proposed 2 model of DNN, have made excellent classification compared to both later models. $100 \%$ samples of $1.75 \mathrm{~m}$ layer have been accurately classified while more than $95 \%$ samples of the other two classes, $0.25 \mathrm{~m}$ and $0.75 \mathrm{~m}$, have been also correctly predicted. The total number of misclassified samples has been 20 samples. The results has shown that vertical positioning DNN model has outperformed other models with the lowest classification rate. $\mathrm{KNN}$ is the second best performing model. Finally, SVC has performed very badly.

Using multi-layered fingerprint database, we have demonstrated how the proposed 3D model has outperformed KNN and SVM. We have accurately implemented this model to our indoor environment and illustrated how this can be implemented.

\section{CONCLUSION}

Throughout this paper, we have introduced the knowledge plane to provide positioning as a service for the 5G IoT network in an indoor environment. We have also demonstrated how this can be implemented in the use case of positioning as a service for complex building. This concept can be also implemented to other smart city use cases such as stadiums, malls navigation systems and indoor AGV control. As for future work, BLE and Wifi data fusion positioning will be implemented to the data plane of this architecture.

\section{ACKNOWLEDGMENT}

This work is supported by HORIZON2020 H2020-MSCARISE-2016 European Framework Program, the SONNET project Consortium, Sigint Solutions Ltd, and London South Bank University. Self-Organization toward reduced cost and energy per bit for future Emerging radio Technologies with contract number 734545 Program.

\section{REFERENCES}

[1] G. M. D. T. Forecast, "Cisco visual networking index: Global mobile data traffic forecast update, 2017-2022," Update, vol. 2017, p. 2022, 2019.

[2] G. A. W. Group. (2017, Dec.) View on 5g architecture. 5GPPP. [Online]. Available: https://5g-ppp.eu/wp-content/uploads/2018/01/5GPPP-5G-Architecture-White-Paper-Jan-2018-v2.0.pdf

[3] M. R. Nowicki and P. Skrzypczyński, "A multi-user personal indoor localization system employing graph-based optimization," Sensors, vol. 19 , no. 1 , p. $157,2019$.

[4] L. Kanaris, A. Kokkinis, A. Liotta, and S. Stavrou, "Fusing bluetooth beacon data with wi-fi radiomaps for improved indoor localization," Sensors, vol. 17, no. 4, p. 812, 2017.

[5] J. Park, Y.-J. Kim, and B. K. Lee, "Passive radio-frequency identification tag-based indoor localization in multi-stacking racks for warehousing," Applied Sciences, vol. 10, no. 10, p. 3623, 2020.

[6] B. Gro $\beta$ windhager, M. Stocker, M. Rath, C. A. Boano, and K. Römer, "Snaploc: An ultra-fast uwb-based indoor localization system for an unlimited number of tags," in 2019 18th ACM/IEEE International Conference on Information Processing in Sensor Networks (IPSN). IEEE, 2019, pp. 61-72.

[7] I. I. Immoreev and P. G. S. D. V. Fedotov, "Ultra wideband radar systems: advantages and disadvantages," in 2002 IEEE Conference on Ultra Wideband Systems and Technologies (IEEE Cat. No.02EX580), 2002, pp. 201-205.

[8] C. Mieth, P. Humbeck, and G. Herzwurm, "A survey on the potentials of indoor localization systems in production," in Interdisciplinary Conference on Production, Logistics and Traffic. Springer, 2019, pp. $142-154$.

[9] A. Boukerche and R. W. Coutinho, "Crowd management: The overlooked component of smart transportation systems," IEEE Communications Magazine, vol. 57, no. 4, pp. 48-53, 2019.

[10] Z. Li, X. Zhao, F. Hu, Z. Zhao, J. L. C. Villacrés, and T. Braun, "Soicp: A seamless outdoor-indoor crowdsensing positioning system," IEEE internet of things journal, vol. 6, no. 5, pp. 8626-8644, 2019.

[11] OpenFlow Switch Specification, 1st ed., Open Networking Foundation, Jun. 2012.

[12] OpenDaylight, "Documentation guide - opendaylight documentation magnesium documentation," https://docs.opendaylight.org/en/stablemagnesium/documentation.html, (Accessed on 05/22/2020).

[13] F. Chollet, Deep Learning with Python, 1st ed. USA: Manning Publications Co., 2017.

[14] A. Kokkinis, A. Paphitis, L. Kanaris, C. Sergiou, and S. Stavrou, "Demo Physical and network layer interconnection module for realistic planning of iot sensor networks," 2018.

[15] GNS3, GNS3 documentation, https://docs.gns3.com/, (Accessed on 05/13/2020).

[16] L. Kanaris, A. Kokkinis, G. Fortino, A. Liotta, and S. Stavrou, "Sample size determination algorithm for fingerprint-based indoor localization systems," Computer Networks, vol. 101, pp. 169-177, 2016. 